\title{
The Atkins diet - bad fat and the cardiovascular system
}

\author{
Christopher Wright* \\ Red Pharm, Cold Meece Estate, Cold Meece, Staffordshire, ST15 0SP, UK
}

\begin{abstract}
Hypertension is estimated to affect 970 million people worldwide and there are a number of pharmaceutical treatments that physicians can prescribe to manage elevated blood pressure. Lifestyle changes can also positively affect blood pressure, which potentially avoids the need for drugs and their potential side effects. This means, due to moderate effects, a much larger population can be potentially reached. This article discusses the results from a study that assessed the effects of dietary vitamins and dairy fats on the cardiovascular system.
\end{abstract}

\section{Introduction}

When it comes to a healthy, balanced diet, The American Heart Association (AHA) recommends the consumption of '...fruits, vegetables, whole grains, beans, legumes, fish, skinless poultry, nuts, and fat-free/low-fat dairy products, and limits sodium, saturated fat, red meat and added sugars' [1,2]. However, other recommendations, notably the Atkins diet, state that 'saturated fat, particularly in the absence of high carbohydrate intake, is not dangerous to human healthon the contrary, when balanced with mono and poly-unsaturated fats in a controlled carbohydrate dietary environment, saturated fat may actually have real and measurable benefits in a number of different arenas [3]. Whilst the Atkins low carb diet is synonymous with stories of weight loss, the recommendation to consume high levels of total fat and saturated fat does not always sit comfortably with everyone as their consumption is commonly related to detrimental health effects. Indeed, the AHA recognises this conflicting information and states [4]:

There's a lot of conflicting information about saturated fats. Should I eat them or not?

The AHA Association recommends limiting saturated fats - which are found in butter, cheese, red meat and other animal-based foods. Decades of sound science has proven it can raise your "bad" cholesterol and put you at higher risk for heart disease.

The more important thing to remember is the overall dietary picture. Saturated fats are just one piece of the puzzle. In general, you can't go wrong eating more fruits, vegetables, whole grains and fewer calories.

A small study published in the European Journal of Clinical Nutrition [1] may hold some insights on the acute intake of low and high saturated dairy fat as it aimed to assess the acute autonomic effects of vitamins and fats in male smokers. Autonomic effects were assessed from changes in baroreflex sensitivity (BRS) and were derived from recordings of arterial blood pressure from the right arm where radial arterial waveforms were acquired for 30-seconds. From these recordings, systolic blood pressure (SBP), pulse interval (the duration between heart beats and heart rate can be calculated as ([1000/pulse interval] x 60 beats per min)) and BRS calculated. Physiologically, BRS is a marker of circulatory autonomic control and has been shown to decline with age and arterial stiffening of the vascular wall [1].

\section{Exploring the effect of fat on the cardiovascular system}

This study recruited thirty smoking subjects of which 26 were entered into a randomised, placebo-controlled, cross-over trial that assessed the acute cardiovascular effects of either placebo (cellulose in capsules), moderate dose of vitamins or a high dose of vitamins consumed on separate visits, with a low ( $50 \mathrm{ml}$ of milk; $5 \mathrm{~g}$ of fat) or high saturated fat challenge $(50 \mathrm{ml}$ of whipping cream; $50 \mathrm{~g}$ of fat). The objective of the study was to assess the anti-oxidative effect of vitamins in smokers thus enabling the assessment of vitamins against a background of increased oxidative stress. In this study, a fat challenge was incorporated into the study protocol as high-fat meals reportedly impair vascular reactivity and BRS; hence, it was used (in the case of whipping cream) to evoke a more marked effect of in the study participants. However, the statistical analysis also enabled the assessment of individual interventions and so from the results it was possible to compare the individual effect of whipping cream (higher saturated fat/lower carbohydrate content; Table $1[5,6]$ ) and milk (lower saturated fat/higher carbohydrate content) on systolic blood pressure and pulse interval. Measuring raised systolic blood pressure (and arterial pressure per se) is important as it may indicate increased arterial stiffness which can damage arteries and leave them less elastic (i.e. atherosclerosis). Pulse interval is needed for the measurement of BRS and therefore important in the assessment of overall cardiovascular health.

\section{The effect of fat on the cardiovascular system}

Four hours after the single ingestion of milk or whipping cream, systolic blood pressure and pulse interval were measured to assess their acute effects on the cardiovascular system. Table 2 shows the effect of milk and whipping cream on systolic blood pressure, pulse interval and BRS. Results clearly show whipping cream increased mean systolic blood pressure $(+2.1 \mathrm{~mm} \mathrm{Hg})$ and decreased pulse interval $(-29.2$ milliseconds or +2 beats per minute) with no effect on BRS.

${ }^{*}$ Correspondence to: Christopher Wright, BSc, MBA, PhD, Red Pharm, Cold Meece Estate, Cold Meece, Staffordshire, ST15 0SP, UK, Tel: 447967230155; E-mail: ciwright26@hotmail.com

Received: July 17, 2018; Accepted: August 08, 2018; Published: August 13, 2018 


\section{The effect of fat on the ageing cardiovascular system}

A post-hoc analysis was conducted on the same dataset to assess the effect of fat on an ageing cardiovascular system. The results of this research were presented at the Summer Meeting of the Nutrition Society [7]. In the study population, the age of the participants ranged between 19 and 45 years of age and a subgroup analysis was conducted to determine if the effect of low and high saturated fat ingestion varied with age (plotted in Figure 1).
Linear plots showed no relationship between age and BRS in the low saturated fat group $(\mathrm{r}=+0.08, \mathrm{P}=0.70$; Figure 1, top panel), but a negative correlation was found in the high saturated fat group $(\mathrm{r}=-0.45$, $\mathrm{P}=0.02$ ). A difference that was principally observed in the oldest age group (aged 40 to 49 years of age) where BRS was statistically ( $\mathrm{P}=0.006$; Figure 2) lower in the high saturated fat group (6.77 vs. $11.54 \mathrm{~ms}$ $\mathrm{mmHg}^{-1}$; high vs. low saturated fat, respectively).

Table 1. Nutritional facts for $100 \mathrm{ml}$ of milk and whipping cream $[5,6]$

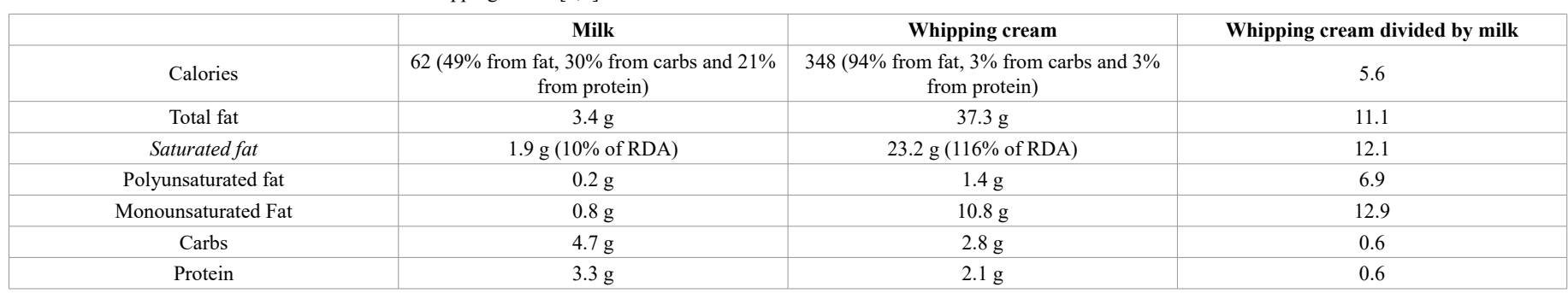

RDA, recommended daily allowance

Table 2. The independent effect of milk and whipping cream on systolic blood pressure, pulse interval and baroreflex sensitivity

\begin{tabular}{|c|c|c|}
\hline Parameter & Milk & Whipping cream \\
\hline SBP $(\mathrm{mm} \mathrm{Hg})$ & $123.4 \pm 0.9$ & $125.5 \pm 0.9$ \\
\hline Pulse interval $(\mathrm{ms})$ & $934.3 \pm 12.1$ & $905.1 \pm 11.9$ \\
\hline Baroreflex sensitivity $\left(\mathrm{ms} \mathrm{mmHg}^{-1}\right)$ & $12.4 \pm 1.2$ & $11.8 \pm 1.1$ \\
\hline
\end{tabular}

Values reported as mean \pm standard error of the mean

\section{Low saturated fat}

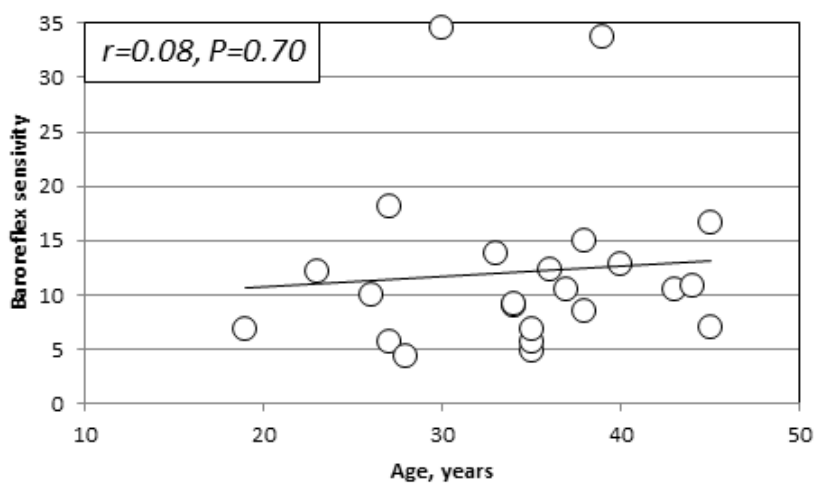

High saturated fat

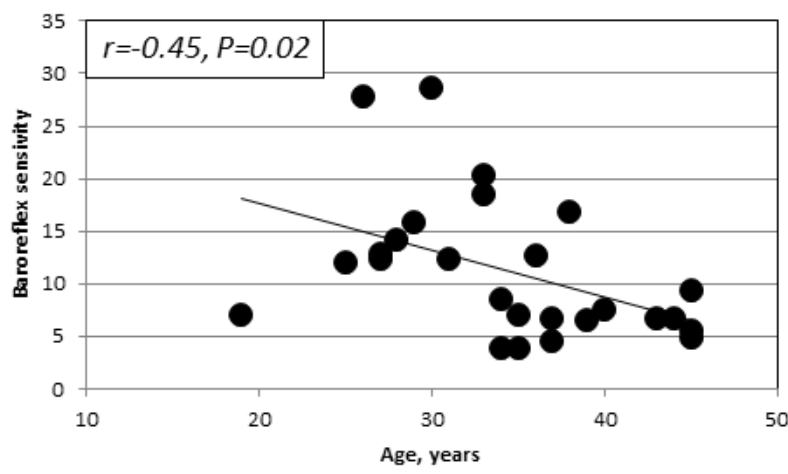

Figure 1. The effect of low (milk - top panel) and high saturated fat (whipping cream - bottom panel) on the relationship between age and BRS 


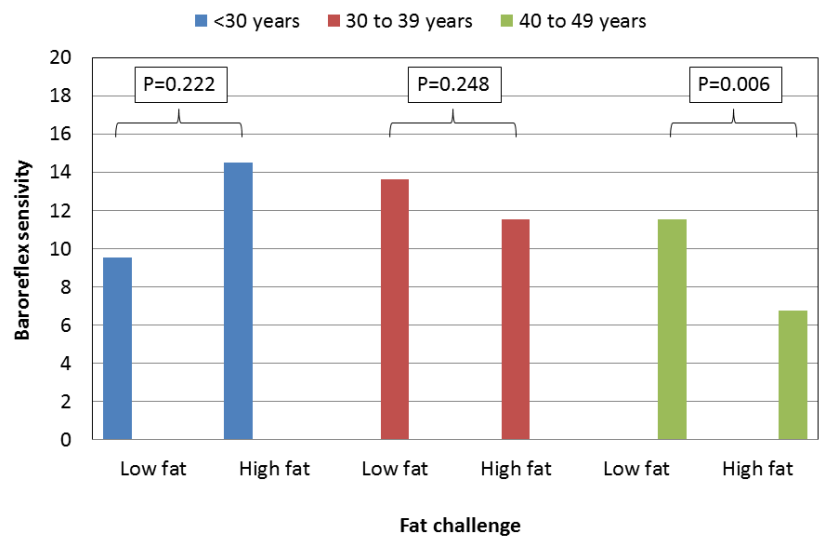

Figure 2. Comparisons of low and high saturated fat groups by age group

\section{Conclusions}

The primary findings from this study show that in male smokers the acute ingestion of a meal that is high in saturated fat and low in carbohydrate, as is commonly recommended in diets such as Atkins, leads to small and detectable changes in both blood pressure and heart. This was observed after a single meal (in this case $50 \mathrm{ml}$ of whipping cream) and within four hours of ingestion. This effect was not observed in the low saturated fat group. The findings highlight the need for careful monitoring of blood pressure and heart rate as repeated ingestion of high saturated fat meals over long periods of time could have a negative effect on individual health which was most evident in older (and smoking) individuals, as indicated by the marked reduction in BRS - an indicator of effective autonomic control of blood pressure and heart rate.

\section{References}

1. Wright CI, Ruediger H, Kroner CI, Janssen BJ, Draijer R (2009) Acute autonomic effects of vitamins and fats in male smokers. Eur J Clin Nutr 63: 246-252.

2. American Heart Association website. Suggested servings from each food group. Source: www.heart.org/HEARTORG/HealthyLiving/HealthyEating/HealthyDietGoals/ Suggested-Servings-from-Each-Food-Group_UCM_318186_Article.jsp\#. WUPnfE2GPIU

3. Aktins website. Atkins position on saturated fat. Source: www.atkins.com/how-itworks/library/articles/atkins-position-on-saturated-fat

4. American Heart Association website. Source: https://healthyforgood.heart.org/Eatsmart/Articles/Saturated-Fats

5. Fatsecret for milk. Source: www.fatsecret.com/calories-nutrition/generic/milk-cowsfluid-whole?portionid $=1136439$ \&portionamount $=100.000$

6. Fatsecret for whipping cream. Source: https://www.fatsecret.com/calories-nutrition/ usda/whipping-cream?portionid $=1137010 \&$ portionamount $=100.000$

7. Wright CI, Ruediger H, Draijer R (2015) With age spontaneous baroreflex sensitivity diminishes after acute fat ingestion. Proceedings of the Nutrition Society 74: E357.

Copyright: ( 2018 Wright C. This is an open-access article distributed under the terms of the Creative Commons Attribution License, which permits unrestricted use, distribution, and reproduction in any medium, provided the original author and source are credited. 